\title{
A Comparative Pilot Study of Historical Artifacts in a CAVE Automatic Virtual Reality Environment Versus Paper- Based Artifacts
}

Sujan Shrestha University of Baltimore

Baltimore, MD 21201, USA

sshrestha@ubalt.edu

J oyram Chakraborty

Towson University

Towson, MD 21251, USA

jchakraborty@towson.edu
Mona A. Mohamed

Towson University

Towson, MD 21251, USA

mmohamed@towson.edu

\begin{abstract}
The purpose of this research is to synthesize and transform real world physical environments (PE) into a CAVE automatic virtual reality system (CAVE) by using three-dimensional (3D) models of cultural and historical artifacts. 3D models are often used in many applications including visualizations and digital preservation. Virtual reality is used to improve perception and sensation and to better understand products and environments for studying human factors and behaviors. As a pilot study, we developed and prototyped a customizable 3D physical environment using historical data and archives into an interactive CAVE virtual reality (VR) system. We then conducted a study of user preferences using pretest and post-test questionnaires of the CAVE versus paper-based artifacts.
\end{abstract}

Author Keywords

CAVE automatic virtual reality, 3D modeling, 3D visualization, Human Computer Interaction, UserCentered Design, Human Factors

\section{ACM Classification Keywords}

Human Factors, User-Centered Design, Modeling, Games, Three-Dimensional Displays, Virtual Reality, 
Computer Vision, Interactive Systems, Information System Education

\section{I ntroduction}

We are on the brink of losing many cultural heritage sites around the world. Man-made and natural disasters are playing a role in this destruction. For example, the seven monuments of Kathmandu valley in Nepal were destroyed in the April 2015 earthquake. (UNESCO to assess the impact on Nepal's cultural heritage of the devastating earthquake. (n.d.). Retrieved April 27, 2015.) Similarly, the Temple of Bel in Palmyra, Syria was destroyed in September 2015. (Director- General Irina Bokova expresses consternation at the destruction of the Temple of Bel in Palmyra. (n.d.). Retrieved September 1, 2015.) These recent destructions remind us of the vulnerability and the need for research in the area of preservation, which embodies human aspiration through history. Preserving and sustaining cultural heritage sites such as these are critical and remains our fundamental responsibility for current and future generations.

Although major institutions across the globe have established preservation programs through libraries, archives, and various national initiatives they are limited to traditional materials in paper, microform, photographic and audio-visual formats. While there are numerous emergences of these efforts partly due to these institutional portals, the documentation process has been slow and expensive (Hedstorm, 1998). Preservation in digital humanities are challenging, largely experimental and yet still untested (Hedstorm, 1998). The presented research addresses these untested areas in the digital and information technology that can be effective and efficient by understanding (a) user preference of color/ pattern, shapes, objects while navigating and locating historical information in a 3D interactive CAVE environment. Such visual display may provide (b) positive user experience of finding historical artifacts.

The contribution of this paper is to find an innovative solution for developing interactive, customizable 3D physical environments of cultural and historic artifacts into a projection-based CAVE automatic virtual reality environment (CAVE). CAVE is a rear-screen projection system that displays $3 D$ environment. The study reports the findings of a comparative study of user preferences of CAVE versus paper-based artifacts. Our initial design and development process started with the preliminary study of a geographical area of Nepal where historical architectures and artifacts were destroyed by the 2015 earthquake. Once we identified sustainable opportunity to preserve cultural and historic landmark/ artifacts, which had the potential of benefiting stakeholders across many scientific and local communities. We identified and differentiate users' patterns of behavior by analyzing, comparing and observing user performance during the experimental period. Observation and comparison of user performance in the PE and VR may better help understand how to best use the technology and develop prototypes for studying human interactions and behaviors (Chen et al. 2014). The rest of the paper is presented as follows. We present a brief literature review of the status of the field of research followed by the methodology we employed. This includes the experimental setup and study design. We then present the findings and analysis of our study. We conclude the paper with a review of the objectives as well as a vision for the next steps in our continued research.

\section{Literature Review}

Modern science, digital, and virtual reality technology today brings an array of possibilities in the area of 
digital preservation of cultural heritage and historical artifacts that have been designed through studies of the human interaction of technologies. The presented pilot study explores a way of finding innovative solutions in converting and sustaining cultural and historical artifacts of a physical environment into a customizable 3D virtual space that can be studied for user preference. It can also be developed as a tool for accuracy and authenticity in the field of digital preservation. Customizable three-dimensional (3D) model refers to the visualization and transformation of a physical environment (PE) into an interactive digital environment (Cruz et al. 1993).

Much of the cultural content documented today is through photo imaging and scanning. Like any medium, this process of documentation has impacted the representation of history and time through interpretation and cultural awareness. Although, the use of virtual reality in archaeology and art history seems like a tangible path, constructing and digitally preserving cultural and historical artifact is a challenging task (Stanco et al. 2011, Chakraborty et al. 2008). Methods of preservation, cost, meta-data and archiving techniques need careful analysis for historical accuracy (Lazinger, 2001). In recent years, the technological development in virtual reality (VR) has provided an ideal platform to transform physical environments into reliable and accurate representations with the use of these sustainable technologies. The potential to unleash new innovations and discoveries from these platforms can be significant and meaningful to scientific communities.

While developing a prototype application, several design approaches can be applied. Several studies have pointed to the technical and operational success of the user-centered design approaches (Jagne, 2004, Mitchell et al. 2004, McCurdie, 2012; Stewart et al. 2013). By engaging the end user from the beginning, we were able to conduct required analysis towards ensuring a usable tool thereby increasing chances of user acceptance. In addition, this approach has helped us document user preferences for any iterative changes as well and future studies.

\section{Methodology}

Participants

Institutional Review Board (IRB) permission was approved at a major university in the United States to carry out this study. Fourteen students were recruited from various classrooms through advertisement across the campus. All fourteen student participants were asked to fill out the consent form followed by a pre-test questionnaire containing questions pertaining to demographics, gaming background and exclusion criteria using a personal computer. The demographics included gender, race/ethnicity, and education. The gaming background included the participant's level of experience, preferred game platforms, and controllers. Exclusion criteria included reported history of seizure, dizziness, disorientation and discomfort while playing video games and exposure to visual stimuli in the VR environment.

\section{CAVE Setup}

The CAVE environment presented in this study was developed using a low-cost hardware and equipment using a computer lab. The lab serves as a research hub for interdisciplinary study in games and media and uses the CAVE system to visualize game interfaces and game contents. The Lab is equipped with immersive, visualization and interactive game technologies. The CAVE VR display screen was created in $8 \mathrm{ft}$. $x 6 \mathrm{ft}$. three-faced walls with six 3D rear short throw projectors. Each 3D projector (NEC M352WS short throw) had maximum 5000 lumens with the 1080p high-definition (HD) display with $70 \mathrm{~Hz}$ of refresh rate with projected wall area of $3 \mathrm{ft} \times 4 \mathrm{ft}$. The distance from the projector to the screen was approximate $3 \mathrm{ft}$. The 
distance between the user and the projected screen was approximately $3 \mathrm{ft}$ in all three directions.

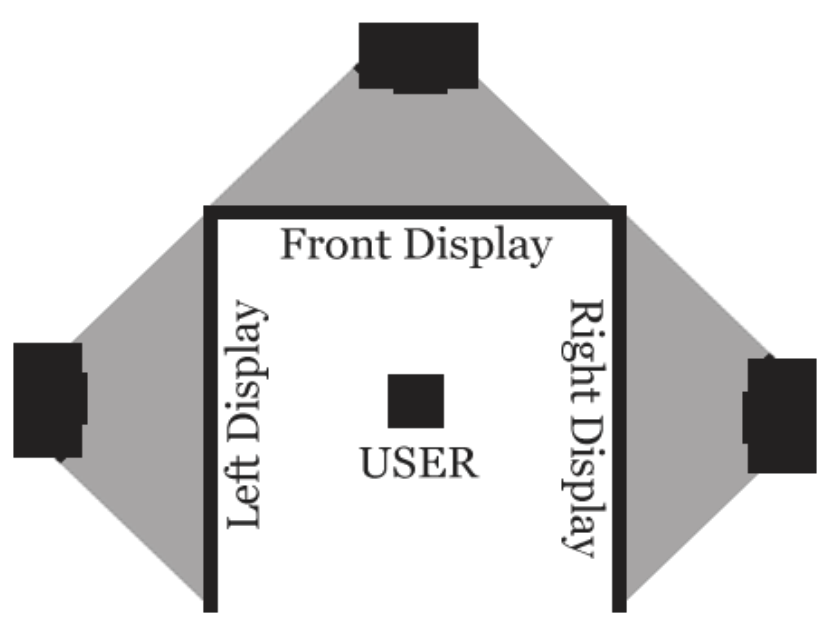

Figure 1: A schematic view of three screens CAVE Automatic Virtual Reality System. The user location from the display screen is approximately $3 \mathrm{ft}$ from all three screens. The

diagram also shows the rear screen short throw projectors.

The 3D models for the virtual environment were developed using Autodesk 3D Studio Max. The textures were developed in the Adobe Photoshop. Unity3D game engine was utilized to develop all interactive contents. The MiddleVR for Unity3D software development kit was used as an intermediate role of connecting host workstation and six remote workstations in the CAVE system. The visual display was generated by a host workstation (Dell 7810 with Intel quad-core Xeon processor, 4GB NVidia Quadro 4400 graphics processor with 64GB RAM). All 3D models and environments were put together using Unity3D game engine. After 3D environments were compiled, we used programming languages ( $\mathrm{C} \#$ and JavaScript) to create an interactive experience. The input device in the CAVE system was a Xbox 360 wireless controller and an NEC active stereoscopic glass. The final product was exported into the MiddleVr software package that allowed us to distribute the projection cluster of the visual display of the 3D historical artifacts into the CAVE environment. The CAVE rear projection display was set up using two projections on each wall. The total of six projectors was utilized to create VR experience. Each projector was connected to a single Dell workstation with Intel 17 processor with 2GB NVidia Quadro 3300 graphics card with 16GB RAM. All six workstations in the CAVE were connected through a network adapter to the host workstation.

We used historical archives and documentation to develop this experimental prototype. Our physical environment site "Bungamati" in the Kathmandu Valley, Nepal, which is historically known as "Rato Machendranath" temple area that dates back to 6th century A.D. was destroyed by the 2015 earthquake Donatella Lorch, Special for USA TODAY: Voices: Like post-earthquake Nepal, Red God is in limbo. (n.d.). Retrieved January 5, 2016.). We documented historical data of images and manuscripts of the surrounding area and developed a prototype of a "Virtual Bungamati". Our development process included customizable and modular 3D models created in the Autodesk 3D Studio Max. Texture maps were developed using photo editing software Adobe Photoshop. The final 3D models were exported into the Unity3D game engine. Programming language $\mathrm{C \#}$ and JavaScript were used and Xbox 360 controller was mapped to interact with the CAVE environment. 


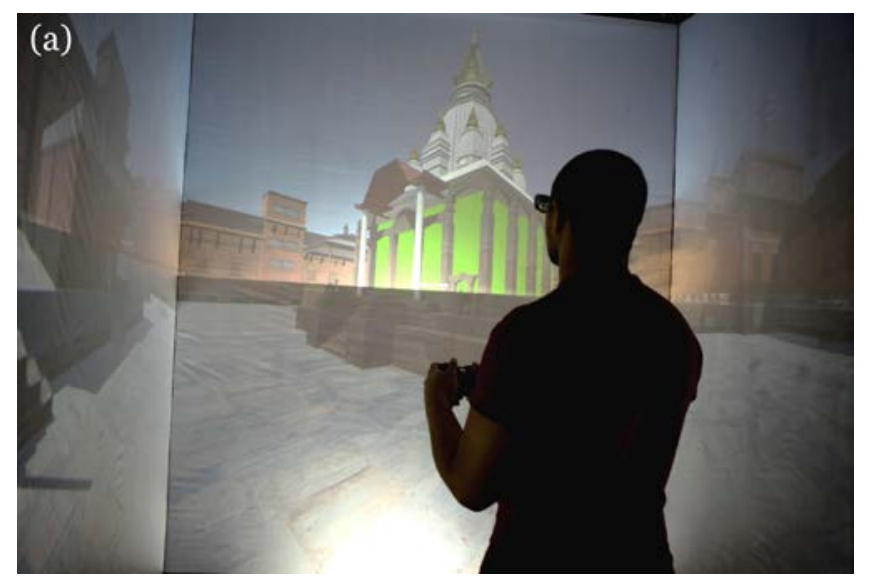

Figure 2: (a) A participant is interacting with 3D models of historical artifacts in a CAVE setup. The highlighted area is the active and interactive selection in the virtual environment.

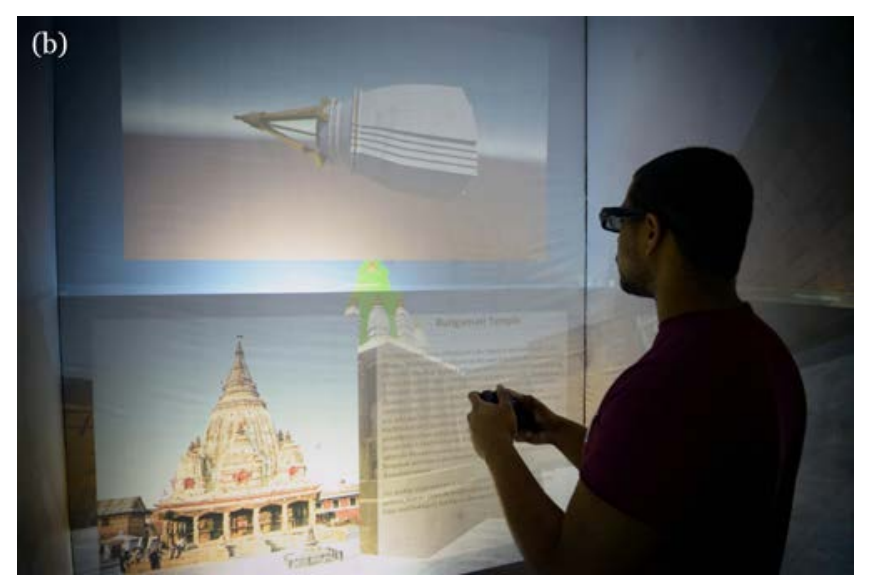

Figure 3: (b) A participant is retrieving information through selected 3D models of historical archives and artifacts from virtual environment of "Bungamati" in Nepal.

\section{Experimental Design}

We compared user preferences between two mediums, VR versus paper-based artifacts. Each participant interacted with both medium and answered some basic questions regarding the content found in them as well as their experiences. As a first step, consent to participate was collected after which every participant was briefed about the study and the potential outcomes. To randomize the experiment, we switched the order in which the participants engaged with the medium i.e. VR first and then paper-based or vice versa. Pre-test questionnaire data was then collected from each user to document their demographics and computer and gaming behaviors. For participants using the VR equipment first, time was given to familiarize themselves with the CAVE setup (Figure 1) while participants using paper-based artifacts first were allowed to get started with the study. Upon completion of each setup (VR or paper-based first), the users were asked to switch to the alternate artifact to carry out the same tasks.

For the CAVE setup, participants were asked to navigate and locate color/ pattern, objects and historical architectures. Users were able to locate and identify these solutions to these questions by interacting with the 3D virtual environment. For the CAVE setup, the historical artifacts were interactively identified by sections of images/ manuscripts, texts and modular three-dimensional historical artifacts. The images and manuscripts were scanned or photographs and placed as reference images from the physical environment. The text-based descriptions were used as supporting documents that were retrieved from the manuscripts and translated in some instances. The three-dimensional modular models of the physical historical artifacts were designed and developed in the lab using high-end computers. The models were identical to the historical artifacts. Users were allowed to interact and rotate the models of historical artifacts which allowed them to retrieve information through text and image through the interface. 
For the paper-based experiment, users were given the liberty to seek answers to the same questions using color printouts of historical manuscripts. Using surveys, user preferences based on each participant's interaction with both sets of equipment were collected from each participant after both setups were tested. Post-test questionnaire contains 10 questions. The data was collected using a 5-point Likert Scale where 1 is easy and 5 is hard.

In this exploratory pilot study, we used 14 student participants as a convenient sample. It gave us an insight and challenges into design changes in the study as well as the interface that must be carried out to improve usability in future studies.

\section{Post-test questionnaires}

Please rate the general ease of use with the virtual reality system.

Please rate the ease of use on finding color/pattern information using the virtual reality system.

Please rate the ease of use finding color/pattern information in the book.

Please rate the ease of use finding shapes/objects in the virtual reality system.

Please rate the ease of use with finding shapes/ objects in the book.

Please rate the overall ease of finding information using the virtual reality system.
Please rate the ease of navigation finding overall information in the book.

If given an architecture development assignment, which tool would be helpful?

Which tool would be faster in finding information you are looking for?

Which tool would be interesting in studying cultural artifacts?

Table 1: Post-test questionnaire

\section{Demographics}

\begin{tabular}{|l|l|}
\hline Gender & $93 \%$ Male \\
\hline $\begin{array}{l}\text { Race/ } \\
\text { Ethnicity }\end{array}$ & $\begin{array}{l}64 \% \text { White/ Caucasian, 21\% African } \\
\text { American }\end{array}$ \\
\hline Education & $80 \%$ Bachelor's degree \\
\hline Game Play & $67 \%$ Play over 3 hours \\
\hline $\begin{array}{l}\text { Game } \\
\text { Controller }\end{array}$ & $43 \%$ Handheld \\
\hline
\end{tabular}

Table 2: Demographics data containing Gender, Race, Education, and the game preference of play and controller 
t-test analysis of user preferences

\begin{tabular}{|c|c|c|c|}
\hline User ID & VR Color & VR Shape & VR Overall \\
\hline 1 & 1 & 1 & 1 \\
\hline 2 & 1 & 1 & 1 \\
\hline 3 & 4 & 5 & 3 \\
\hline 4 & 2 & 3 & 1 \\
\hline 5 & 1 & 1 & 1 \\
\hline 6 & 3 & 2 & 2 \\
\hline 7 & 1 & 1 & 1 \\
\hline 8 & 2 & 2 & 2 \\
\hline 9 & 1 & 1 & 2 \\
\hline 10 & 1 & 1 & 1 \\
\hline 11 & 1 & 1 & 1 \\
\hline 12 & 1 & 2 & 2 \\
\hline
\end{tabular}

\begin{tabular}{|c|c|c|c|}
\hline 13 & 2 & 1 & 1 \\
\hline 14 & 1 & 1 & 2 \\
\hline Results & 0.02 & 0.04 & 0.06 \\
\hline
\end{tabular}

Table 3: Results of t-test * only significant results are shown

\section{Analysis}

All participants reported playing video games regularly since all the participants were over 18 and millennial generation, this might be considered as a limitation of the study as other generations are not tested. Seizure and dizziness that were part of the exclusion criteria have no effects on VR factors since none of the participants reported any adverse effect. $57 \%$ of the participants reported having no experience with the VR and $36 \%$ reported having used VR once. The gender makeup of the study was $93 \%$ male. $64 \%$ of the participants identified themselves as White/Caucasian and $21 \%$ as African American. All the participants reported having some form of video gaming experience with most indicating approximately $2-3$ hours a day of video game plays. The participants did not mention a specific game preference. However, the majority of the participants did indicate a preference for handheld game controllers.

The results from data collected from the 14 participants indicated that only a small number of participants found it easier to search for details and solve problems better using VR. $50 \%$ of the participants indicated some skepticism towards the CAVE as a tool to solve complex problems. This finding is consistent with the technology acceptance model literature, which suggests initial resistance towards new technologies. The Likert scale 
data of user preferences using VR for all the participants was analyzed for significance using t-tests. The results of the t-test showed significance at 95\% confidence $(a<0.05)$ for VR Color, VR Shape, and VR Overall only. This implies that the participants found the VR tool to be easier to use when carrying out searches for color patterns, shapes and historical information in this study.

\section{Conclusion}

In this paper, we carried out a pilot study of human factors in the development and prototyping of a customizable 3D physical environment using historica data and archives into an interactive CAVE VR system. We compared human factors and behaviors by analyzing pretest and post-test questionnaires of user preferences in the CAVE versus paper-based artifacts to digitally recreated historical sites from the post-2015 earthquake in Nepal. Our analysis revealed some challenges with the user acceptance of the new technology. However, the findings also indicated users ease of use finding details in the artifacts using the VR setup.

\section{Limitations of the study}

Although the findings in this study showed some significant preferences for VR as a tool in digitally preserving at-risk cultural artifacts, the results are not generalizable owing the small sample size. However this was not the objective of the study. The goal of this study was to develop a comparative pilot study that uses an innovative solution in historical and cultura preservation. Clearly, further studies using larger sample sizes are required to extend the literature further.

\section{Future Works}

Digital preservation is a challenging and daunting task when visualizing an archival footage of a site with limited documentation. Despite the richness and complexity of the above pilot study, historical data collection of this nature could hold a significant and noble resource for the study of historical artifacts and cultural heritage for people around the world.

Preservation in digital humanities are challenging, largely experimental and yet still untested (Hedstorm, 1998). Very little effort has been put in for the collection of destroyed artifacts and little steps that have been taken to keep the archives concurrent in Nepal after the earthquake. Nepal's current economy and continuing political crisis are the two major factors that prevent these cultural heritage sites to be archived. This pilot study of virtualizing cultural heritage is an ideal solution that can be accomplished with minimal disturbance to the ancient archeological sites around the world. This method will also allow the preserved artifacts to be accessible worldwide through the world-wide-web.

In the future, field and ethnographic research could help to create broader as well as in-depth understanding of this field. This will expand the scientific inquiry in the preservation of physical environments into the digital domain. The presented research is an incremental step forward in the interdisciplinary research domain of digital preservation and human-computer interaction. It has the potential to serve as a bridge between two disciplines that could significantly benefit from additional research. 


\section{References}

1. Barris, K. 2007. “To Be Virtual ... or Not to Be: Theatre Museum Canada's Travels into the World of Technology", Canadian Theatre Review, (131), 4952.

2. Chen, K. B., Kimmel, R. A., Bartholomew, A. Ponto, K., Gleicher, M. L., \& Radwin, R. G. 2014. "Manually Locating Physical and Virtual Reality Objects", Human Factors: The Journal of the Human Factors and Ergonomics Society, 56(6), 1163-1176.

3. Chen, S. 2001. "The paradox of digital preservation", Computer, 34(3), 24-28.

4. Chu, C.--C. P., Dani, T. H., \& Gadh, R. 1998. "Evaluation of virtual reality interface for product shape designs", Iie Transactions, 30, 7. 629--643.

5. Cruz-Neira, C., Sandin, D. J., \& Defanti, T. A. 1993 "Surround-screen projection-based virtual reality", Proceedings of the 20th Annual Conference on Computer Graphics and Interactive Techniques SIGGRAPH '93.

6. Caffo, R. 2014. “Digital Cultural Heritage Projects: Opportunities and Future Challenges", Procedia Computer Science, 3812

7. Chakraborty, J., Hansen, L., Denenberg, D. A., \& Norcio, A. F. 2009. "Preliminary Findings from a Cross Cultural Usability Study on the Internationalization of User Interfaces", Online Communities and Social Computing Lecture Notes in Computer Science, 162-171.

8. Christou, C., Cameron A., Loscos, C., Dettori, A. 2006. "A Versatile Large-Scale Multimodal VR System for Cultural Heritage Visualization", OpenSample, DOI 10.1145/1180495.1180523.

9. Fresa, A. 2014. "Digital Cultural Heritage Roadmap for Preservation", International Journal of Humanities and Arts Computing Intl J Humanities \& Arts Computing, 8(Supplement), 107-123.

10. Grau, O. 2003. "Virtual art: From illusion to immersion", Cambridge, Mass: MIT Press.
11. Gomez-Lahoz, J., \& Gonzalez-Aguilera, D. 2009. "Recovering traditions in the digital era: The use of blimps for modelling the archaeological cultural heritage", J ournal of Archaeological Science, 36(1), 100-109.

12. Hedstrom, M. 1998 “Digital Preservation: A Time Bomb for Digital Libraries", Computers and the Humanities. 31 (3), pp. 189-202.

13. Ikeuchi, K., Oishi, T., Takamatsu, J., Sagawa, R., Nakazawa, A., Kurazume, R., Okamoto, Y. 2007. “The Great Buddha Project: Digitally Archiving, Restoring, and Analyzing Cultural Heritage Objects", I nternational J ournal of Computer Vision Int J Comput Vis, 75(1), 189-208.

14. Maschner, H. D., \& Schou, C. D. 2013 "Virtualization and the democratization of science: 3D technologies revolutionize museum research and access", 2013 Digital Heritage International Congress (DigitalHeritage).

15. Nan, X. Ziyang, Z. Ning, Z (2014). 2014. "vDesign: a CAVE-based virtual design environment using hand interactions", J Multimodal User Interfaces. 8:367-379. doi: 10.1016/j. procs.2014.10.003

16. Nawa, L. L., \& Sirayi, M. 2014. "Digital Technology and Cultural Heritage Sites in the City of Tshwane", The Journal of Arts Management, Law, and Society, 44(4), 246-257.

17. Repperge, D. W. 2003. "Effects Of Haptic Feedback And Turbulence On Landing Performance Using An Immersive Cave Automatic Virtual Environment (Cave)", Perceptual and Motor Skills PMS, 97(7), 820.

18. Ryan, J. C. 2015. "The Virtual and the Vegetal: Creating a 'Living' Biocultural Heritage Archive through Digital Storytelling Approaches", Globa Media Journal: Australian Edition, 9(1), 49-58.

19. Yan Peng, L. 2011. "Virtual Reality as a Means for Preserving Digital Heritage: Masjid Jamek",

International Journal Of I nterdisciplinary Social Sciences, 5(9), 119-128.

20. Stewart, Osamuyimen T., David M. Lubensky, and Joyram Chakraborty. 2013. "Systems and methods 
for automatically determining culture-based

behavior in customer service interactions", U.S.

Patent

No. $8,478,594$.

21. Stanco, F., Tanasi, D., \& Gallo, G. 2011. “Virtual Restoration of Fragmented Glass Plate Photographs

of Archaeological Repertoires", Virtual Archaeol.

Rev. Virtual Archaeology Review, 2(3), 141

22. Thomsen, J., Woerum, J., Haagensen, $\mathrm{H}$.

"Bungamati 1968, A Survey by Danish Architect

Students", Center for Cultural and Development, Embassy of Denmark.

23. Vilbrandt, C., Pasko, G., Pasko, A., Fayolle, P.

Vilbrandt, T., Goodwin, J. R., Kunii, T. L. 2004.

"Cultural Heritage Preservation Using Constructive Shape Modeling", Computer Graphics Forum,

23(1), 25-41. 